\title{
A Biologically Inspired Model for Signal Compression
}

\author{
Mark D. McDonnell ${ }^{*}$ and Derek Abbott, \\ Centre for Biomedical Engineering (CBME) and \\ School of Electrical \& Electronic Engineering, \\ The University of Adelaide, SA 5005, Australia
}

\begin{abstract}
A model of a biological sensory neuron stimulated by a noisy analog information source is considered. It is demonstrated that action-potential generation by the neuron model can be described in terms of lossy compression theory. Lossy compression is generally characterized by (i) how much distortion is introduced, on average, due to a loss of information, and (ii) the 'rate,' or the amount of compression. Conventional compression theory is used to measure the performance of the model in terms of both distortion and rate, and the tradeoff between each. The model's applicability to a number of situations relevant to biomedical engineering, including cochlear implants, and bio-sensors is discussed.
\end{abstract}

Keywords: lossy compression, quantization, cochlear implants, biosensors, neural coding, bioinspired compression

\section{INTRODUCTION}

Sensory neurons in the nervous system convert external stimuli into electrical and chemical signals, so that information can be transmitted to higher functional centres in the brain. Such neurons can act both independently and in cooperation. ${ }^{1,2}$ Stimuli are often continuously valued variables that can be modeled as analog random signals.

Some neurons, such as in the insect early visual system, represent information by graded (continuously valued) voltages. However, when information is required to propagate over longer distances 'spike trains', or action-potentials are used. ${ }^{3}$ These are short-duration large amplitude pulses. Shortly after each pulse, there is a time period during which no new action-potential can form. This duration, and the duration of the actionpotential, is known as the refractory time.

There are a number of different mechanisms by which action-potentials encode information, such as (i) latency, (ii) rate coding, ${ }^{2}$ (iii) temporal coding, (iv) place coding, ${ }^{4}$ and (v) population coding. ${ }^{5}$ The focus here is on sensory neurons that produce action-potentials that form a rate code representation of a random analog stimulus. This is somewhat analogous to frequency modulation, in that a rate code encodes some quantity via the frequency at which action-potentials are produced. The perspective of direct observations made by an experimenter is used.

From an information-theoretic viewpoint, rate codes necessarily involve the loss of information. The aim here is to show how biological neural rate coding can be described in an information-theoretic manner using lossy compression theory, and in particular, quantization theory. ${ }^{6}$ The eventual goals are that this computational neuroscience approach may lead to improved understanding of the neural code. Understanding how neurons compress information may lead to improved or new forms of biomedical prosthetics, such as cochlear implants.

The remainder of this introduction outlines lossy compression theory, and the neural rate coding paradigm. Sec. 2 discusses how to describe neural rate coding using lossy compression theory. In Sec. 3, the FitzHughNagumo neuron model is used as an illustrative example of how these lossy compression ideas can be applied to neural coding. Sec. 4 contains some speculations on possible biomedical applications where increased knowledge about how neurons compression information could be utilized. Finally, Sec. 5 summarizes the outlined lossy compression approach for understanding sensory neural coding, and lists some future directions for this research.

\footnotetext{
*mmcdonne@eleceng.adelaide.edu.au; phone +61 88303 6296; fax +61 883034360
} 


\subsection{Lossy Compression and Quantization Theory}

Lossy compression involves deliberately 'throwing away' information, in order to reduce the 'cost' of storing or communicating it. Decompressing the result gives data that is different from the original, but is 'close enough' to be useful in some way. Familiar examples include the JPEG and MP3 standards for image and audio compression.

Quantization refers to a specific form of lossy compression: the situation where the information being compressed is an analog 'signal' and the compressed version is a discrete or digital representation of that signal. ${ }^{6}$ Familiar applications where quantization is used include analog-to-digital converter circuits, and the formation of histograms from real-valued data.

There are two conflicting requirements in the design of a quantizer - or indeed any lossy compression scheme: (i) minimizing the loss; and (ii) maximizing the amount of compression. Information theory can assist in designing quantizers that are optimized to meet specified performance requirements, or in analyzing the performance of some quantizer. ${ }^{6}$

Regardless of the application in which quantization is required, the operation can be abstracted to the following model with three main components:

- Encoder: the mapping of a space, $A$, to an index set $I, A \rightarrow I$. E.g. the space is typically $\mathbb{R}^{N}$, and the index set a subset of $\mathbb{Z}^{+}$;

- Decoder: the assignment of a reproduction value to each index of the encoder mapping: the codebook;

- Distortion Measure: a mathematical description of the error incurred by quantizing using a given encoder and decoder.

\subsubsection{Scalar quantizers}

A complete description of a scalar quantizer - i.e. one in which the input is a scalar variable - is given by (i) a set of threshold values that describe the cell boundaries (the encoder); and (ii) a set of reproduction values, that describes all values in a cell (the decoder). The reproduction values are required in order to construct an approximation to the original signal. These two sets together define the quantizer's transfer function, $Q(x)$, which maps a source, $x$, to $Q$ - see Fig. 1.

Consider a scalar quantizer which uses $N-1$ thresholds, with values $\theta_{1}, . ., \theta_{N-1}$. If the output states are labeled as $y=1, . ., N$, the output of the encoder stage can be written as

$$
y(x)=\frac{N+1}{2}+\frac{1}{2} \sum_{i=1}^{N-1} \operatorname{sign}\left[x-\theta_{i}\right], \quad y \in[1, . ., N] .
$$

If the reproduction point corresponding to output state $y$ is written as $Q(y), y=1, . ., N$, then $Q(y)$ is the output of the decoding stage, and $Q(y(x))=Q(x)$ is the transfer function.

Quantization of real-valued quantities can never be perfectly reversed. Reproduction of the original signal will always result in some error signal, however small. This error is known as the distortion, or quantization noise, and is most commonly measured by the mean square error (MSE) between the original signal, $x$, and the reconstructed signal, $Q(x) .{ }^{6}$ The error signal is defined as a function of $x$ as $\epsilon(x)=x-Q(x)$.

Suppose $x$, is a sequence of $i i d$ samples from a continuously valued probability distribution, with probability density function (PDF), $P_{x}(x)$. If $P_{x}(x)$ has support (non-zero values) $x \in[a, b]$, the MSE distortion can be written as

$$
D_{\mathrm{ms}}=\sum_{n=1}^{N} \int_{\theta_{n-1}}^{\theta_{n}}(x-Q(n(x)))^{2} P_{x}(x) d x,
$$

where $\theta_{0}=a$ and $\theta_{N}=b$ are implicit thresholds at the limits of the support of $P_{x}(x) .{ }^{6}$ 


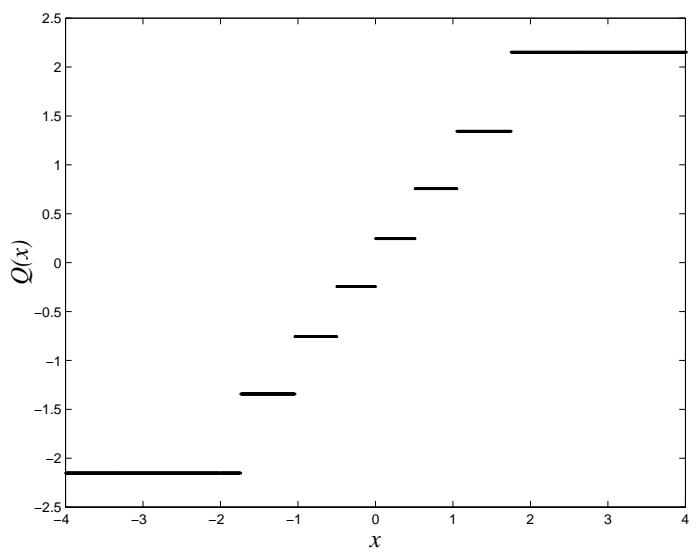

Figure 1. Example transfer function of a quantizer. An input signal (information source), $x$, is converted to a discrete representation, $Q(x)$.

A commonly used measure of a quantizer's performance is its (output) signal-to-quantization-noise ratio (SQNR), which is the ratio of the input signal power to the MSE distortion power. If the input signal has power $\sigma_{x}^{2}$, then this can be expressed as

$$
\mathrm{SQNR}=10 \log _{10}\left(\frac{\sigma_{x}^{2}}{D_{\mathrm{ms}}}\right)
$$

Quantizer design and performance analysis can be assisted by the use of rate-distortion theory ${ }^{7 *}$. This is discussed further below, in the context of neural coding.

For a scalar quantizer, the rate can be defined simply as the number of output states used, $N$, which can be converted to bits as $R=\left\lceil\log _{2}(N)\right\rceil$, where $\lceil\cdot\rceil$ is the ceiling operator. In certain situations, the rate is defined as the output entropy, or mutual information, however here we do not require this. Rate-distortion analysis looks at the tradeoff between rate and distortion - increasing $N$ will decrease distortion, but increase rate and vice versa. A designer wishes to know "what is the minimum rate required to achieve a certain distortion?"

\subsection{Neural Rate Coding: Firing Rates and Stimulus-Response Curves}

Rate code analysis of neural behavior requires an assumption that individual action-potential timings are not important. Instead, neural recordings are characterized by their 'firing rates'-i.e., the average number of actionpotentials observed while a given stimulus intensity, $x$, is fixed for some duration, $T$. Averaging is necessary because the number of action-potentials produced is usually observed to be a random variable, ${ }^{1}$ but as we will see, there is more than one way to average.

Neurons which are assumed to produce rate code action-potentials in response to a stimulus can be characterized by stimulus-response curves. These are plots of average firing rate against stimulus intensity, and are also known as tuning curves. ${ }^{1}$ Stimulus-response tuning curves should not be confused with tuning plots specific to auditory neuroscience, in which the firing rate response of a neuron is plotted as a function of stimulus frequency, rather than intensity.

Stimulus-response curves are obtained empirically by applying a fixed stimulus, $x$, at the input to a neuron, and recording the times at which action-potentials are produced during some duration, $T$. There are several ways in which the term 'firing rate' can be defined. ${ }^{1}$ The two most relevant meanings are now discussed.

\footnotetext{
*In this context, the term rate relates to the amount of compression, rather than a 'per unit time' rate. The term distortion refers to how 'different' the compressed information is to the original information.
} 


\subsubsection{Instantaneous Firing Rates and Inter-Spike-Interval Histograms}

In this analysis paradigm, the timing of individual action-potentials is utilized. Suppose a stimulus, $x$, is held constant for some duration, $T$. The instantaneous firing rate at time $t_{o} \in[0, T]$ is defined as the reciprocal of the duration, $\Delta$, between the last two action-potentials prior to time $t_{o}$. This duration is often called an inter-spike-interval (ISI). It is quite common for ISIs to vary considerably for a fixed stimulus. This means plots of instantaneous firing rate against time can be quite noisy. In order to estimate the probability distribution of the ISIs, histograms of ISIs are often formed-i.e. ISI histograms. The mean value of the measured ISIs provides the average firing rate for stimulus $x$.

If this procedure is repeated for a range of stimulus intensities, the stimulus-response curve can be found as a function of $x$. Since the firing rate response for each value of $x$ is an average, there will be some variance about the mean value. This variance may also be a function of $x$.

\subsubsection{Spike-Counting Firing Rates}

An alternative way of measuring average firing rates is to ignore the individual action-potential timings, and to simply count the number, $n$, of action-potentials that occur during duration, $T$, in response to fixed stimulus, $x$. The average firing rate is then defined as $n / T$ action-potentials per second. This may be repeated for the same $x$ over multiple experiments, and the ensemble average of $n / T$ obtained.

Due to finite refractory times, the number of action-potentials during $T$ has some upper-limit, say $N$. The lower limit is zero, i.e. when no action-potentials occur. Therefore, the spike-count rate is limited to a finite number $(N+1)$ states, and is a quantized representation of the stimulus, $x$.

\subsubsection{Comparing ISI Analysis with Spike-Count Firing Rates}

Formation of firing rates from measured ISIs appears on the surface to consist of entirely analog processing. However, as with all analog processing, noise limits the precision to which experimental measurement of ISIs can be made. Measuring an ISI duration to a high precision implies that such a high precision is important, when in reality - in particular for low SNR stimuli - it is surely not. Furthermore, since rate coding analysis literally provides firing rates in units of 'action-potentials per second,' it is surely better calculated from an integer count, rather than an ISI duration. Given that this count is a quantized representation of a random stimulus, lossy compression theory is an appropriate tool.

Spike-counting is not without its own problems though - in particular the issue of how long should be the duration, $T ?^{1}$ Addressing this is not required for our slightly abstract discussion below.

\section{NEURAL RATE CODING AS LOSSY COMPRESSION}

\subsection{Why Use a Lossy Compression Framework to Describe Neural Rate Coding?}

The task of some sensory neurons and neural populations is to represent, using action-potentials, the amplitude of a time-varying analog stimulus; e.g.

- auditory system: loudness of a sound;

- visual system: velocity of prey.

Because the stimulus amplitude is analog, unless it can be represented to infinite precision some information is lost. In theory, the time between any two action-potentials in a single neuron is an analog quantity that could encode an analog amplitude to infinite precision. However, due to non-zero refractory times, such a coding mechanism is impossible for stimuli with rapidly varying amplitudes. Furthermore, even a tiny amount of random noise in such an encoding would mean infinite precision is lost. This is why empirically produced instantaneous firing rates are very variable.

Due to the fact that information is necessarily lost, coding of stimulus amplitudes via firing rates can be viewed as lossy compression. This should not be viewed as a limitation; an optimized data acquisition system is one which provides only the minimal amount of information required to achieve some goal. This statement 
is formalized in information theory as rate-distortion theory. ${ }^{7}$ To differentiate between this usage of 'rate', and neural firing rates, we will use the terms 'compression rate', and 'firing rate'.

Using a lossy compression framework to describe neural allows analysis of how 'efficient' a sensory neural system is at encoding information. Any such analysis for a specific cell or population can be compared with theoretical limits, as well as with other different cells, populations, species or stimuli. Furthermore, part of the motivation for this work is to study the capability of sensory neurons to operate effectively with very low input signal-to-noise-ratios. ${ }^{8}$ This may lead to bio-inspired or bio-mimetic approaches to lossy compression in artificial low-SNR sensor systems.

\subsection{Lossy Compression Perspective of Rate Coding}

\subsubsection{Populations of Neurons}

Fig. 2 shows an abstract block diagram of the information theory relevant aspects of action-potential generation in a network of parallel sensory neurons. Although this is implicitly a discrete time model, non-zero refractory times mean that it can argued as still being relevant to the realistic continuous time situation - although perhaps with some modifications.

A sample, $x$, of an analog (continuously valued) random stimulus, $X$, with a PDF, $f_{X}(x)$, is 'received' by $N$ neurons. It is assumed that due to spatial separation, all $N$ neurons are subject to iid (independent and identically distributed) additive input noise, $\eta_{i}, i=1, . ., N$, with PDF $f_{\eta}(\cdot)$.

As an example of what is meant by 'received', in the inner ear auditory signals mechanically deflect stereocilia in inner hair cells. These hair cells produce neurotransmitters - in amounts corresponding to the magnitude of deflection - at synapses with $N$ parallel afferent nerve fibres, where action-potentials may be produced, and transmitted to the cochlear nucleus. The 'received' signal is the auditory stimulus that deflects the stereocilia.

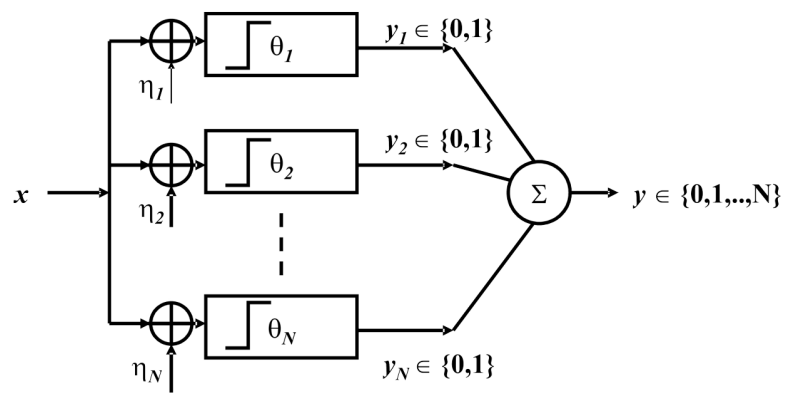

Figure 2. Abstract model of an array of neurons.

Each neuron in the population has a static 'threshold value' $\theta_{i}$ that determines when action-potentials are produced. When the sum of the stimulus, $x$, and noise $\eta_{i}$, is larger than the threshold value of the $i$-th neuron, $\theta_{i}$, an action-potential is produced, i.e. if $x+\eta_{i} \geq \theta_{i}$. If the $i$-th neuron produces an action-potential, then we write its output as $y_{i}=1$. Its output is $y_{i}=0$ otherwise. The overall population output, $y$, is the sum of the $y_{i}$ 's, and represents the total number of action-potentials produced in response to $x$.

The threshold values are assumed to be large enough that frequent spontaneous firing can occur, and that stimuli can be both excitatory and inhibitory. In other words, when the stimulus value, $x$ is well below its mean value, the firing rate approaches zero (no neurons produce action-potentials). When the stimulus is well above its mean, the firing rate approaches saturation (all $N$ neurons produce action-potentials). 


\subsubsection{Individual Neurons}

The population model above can be transformed into a nearly equivalent model for a single neuron. If thresholdadaptation is ignored, this has the consequence that all threshold values are identical. Furthermore, in order to obtain a spike-count, a duration, $T$, during which $x$ is fixed constant needs to be specified. The maximum value of $y$, i.e. $N$, is the maximum number of action-potentials that can occur during $T$. If a constant refractory time, $t_{r}$, is assumed, then $N=T / t_{r}$.

With these caveats in mind, it can be seen that both a population of neurons, and a single neuron can be described in the manner of a scalar quantizer. A similar scenario to this translation from a parallel population to a single neuron appears elsewhere in the computational neuroscience literature. ${ }^{9}$

\subsection{Important Time Scales}

It is very important to note that if the input stimulus is a random variable, then the correlation time, $\tau_{s}$, of the stimulus is a crucial factor in determining approximately how long the duration, $T$, can be for a spike-count to be a meaningful representation of $x$. In turn, since the refractory time also determines $N$, it is clear that three biophysical time-scales must be factored into any lossy compression analysis of neural coding:

1. the refractory time, $t_{r}$;

2. the stimulus correlation time, $\tau_{s}$;

3. the counting time, $T$-biophysically, this would correspond to the 'memory' of the neural system at which action-potentials are received.

For very rapidly varying stimuli - i.e. short correlation times - a single neuron may not be sufficient to encode all relevant changes in stimulus. This means that an ideal condition for the proposed quantization framework to apply to a single neuron is that $t_{r} \ll T \ll \tau_{s}$.

In the event that this does not hold and $T$ must be much shorter, the same performance can be obtained with parallel neurons and a short $T$, as in Fig. 2. However, in this case, unless each neuron has different dynamics, good performance is only achieved if independent random noise acts to randomize the quantization thresholds. This leads to an effect known as suprathreshold stochastic resonance (SSR) ${ }^{10,11}$-i.e. the presence of random noise is required for optimal performance. This can happen because independent random noise acts to effectively distribute threshold values, and a random scalar quantization is achieved.

In fact, SSR can also occur in the single neuron model provided - as assumed - the threshold value remains constant. This is analogous to dithering in a single bit ADC. ${ }^{12}$ In the absence of noise, the action-potential count for a fixed stimulus will either be zero or $N$. In the presence of noise, the expected count is a continuous one-to-one function of $x$. This idea however is not easily investigated in the FHN neuron example below, as the FHN equations in fact act as though the neuron has a dynamical threshold value. Further investigation is left for future work.

\subsection{Compression Rate and Distortion}

For neural coding, it is far from clear what a good distortion measure would be. Therefore, for this preliminary work, the standard mean square error distortion is used. However, the compression rate can be simply defined as the maximum number of action-potentials that can occur during some duration, $T$, i.e. the compression rate is simply $R=T / t_{r}=N$. 


\section{EXAMPLE: A SINGLE FITZHUGH-NAGUMO NEURON MODEL}

\subsection{The FitzHugh-Nagumo Equations}

In this section we discuss the FitzHugh-Nagumo (FHN) model of neural action-potential generation. ${ }^{13}$ This model describes the output voltage signal of a neuron in response to a given stimulus via a pair of coupled differential equations. These equations are a simplification of the Hodgkin-Huxley neuron model. The output signal of a neuron in response to any given stimulus can be simulated by numerically solving the FHN equations.

Many equivalent forms of the equations exist; we use the following form, ${ }^{13}$

$$
\begin{aligned}
& \frac{d X(t)}{d t}=f(X(t))-Y(t)+I(t) \\
& \frac{d Y(t)}{d t}=b(X(t)-\gamma Y(t)),
\end{aligned}
$$

where $f(x)=k x(x-a)(1-x), X(t)$ is the 'voltage' variable that represents the output of the neuron, $Y(t)$ is an auxiliary 'recovery' variable, and $I(t)$ is an input stimulus.

The FHN equations can be extended to incorporate the effects of random noise. For example, if the input current is modeled as subject to additive noise, $\xi(t)$, the equations becomes stochastic differential equations (SDEs). ${ }^{13}$ Using the notation of SDEs, we have

$$
\begin{aligned}
& d X(t)=[f(X(t))-Y(t)+I(t)] d t+\beta d W(t) \\
& d Y(t)=b(X(t)-\gamma Y(t)) d t
\end{aligned}
$$

where $W(t)$ is a continuous-time noise process. If the noise has a short correlation time, the equations need to be solved using special numerical SDE techniques. Otherwise, ordinary methods are sufficient.

The parameters used $\mathrm{in}^{13}$ lead to the following more familiar ODE form of the equations,

$$
\begin{aligned}
& \frac{d x}{d t}=0.5 x(x-0.1)(1-x)-y+I(t)+\beta \xi(t) \\
& \frac{d y}{d t}=0.015(x-0.2 y),
\end{aligned}
$$

with $x(0)=1.1$ and $y(0)=1.1$. These equations can be easily solved numerically as a function of time using standard methods.

The only remaining unknown is the driving current, $I(t)$. The FHN equations do not always act like a neuron. In particular, it is well known that Hopf bifurcations occur in the FHN equations for a driving current that remains constant with time. ${ }^{13}$ This means that there is a range of values of $I$ for which oscillations - i.e. action-potentials - are sustained. Outside this range, the voltage variable decays to a fixed value.

\subsection{Absence of Noise}

Fig. 3 shows the output voltage, $x(t)$, that results after solving the FHN equations given by Eqs. (8) and (9), for an input current $I(t)=1.8$. Each peak in $x(t)$ represents an action-potential at the output of a neuron.

For the parameters used here, action-potentials are sustained if $I \in[0.3,3.3]$. If the input current is chosen from this interval, and held fixed for some duration, $T$, then the number of action-potentials during this interval is a constant. The inter-spike-interval (ISI) is also constant. This means that the firing rate is determined by the time between any two consecutive action-potentials, and theoretically has no variance.

However, the ISI is a function of $I$, and therefore we can numerically calculate an exact tuning curve for the FHN neuron model. This is shown in Fig. 4 , and replicates Fig. $9 \mathrm{C}$ in. ${ }^{13}$ The $x$-axis in Fig. 4 is the input current, and the $y$-axis is the firing rate calculated as the reciprocal of the ISI. 

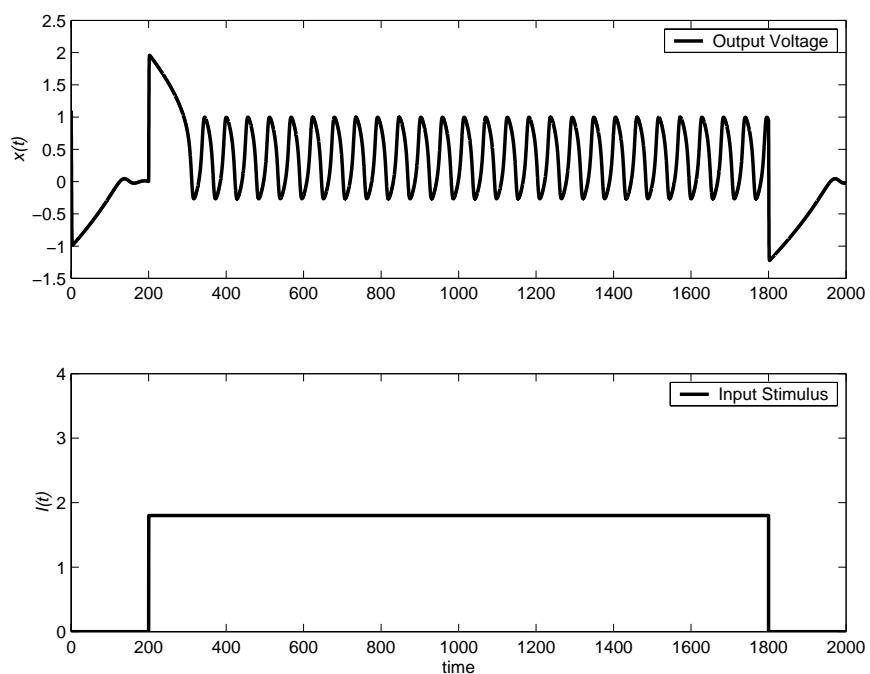

Figure 3. FHN input current and output voltage in the absence of input noise, i.e. $\beta=0$. The stimulus is constant at $I=1.8$. Units are arbitrary.

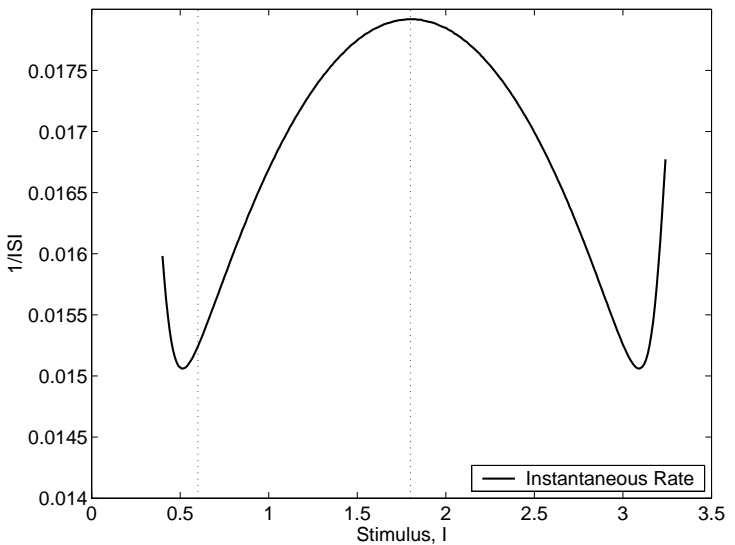

Figure 4. Tuning curve for the FHN neuron model in the absence of noise. The units of $I$ are arbitrary.

\subsection{Presence of Noise}

In reality, the input current is noisy and the ISIs will not remain constant for a constant $I(t)$. As explained, this is why firing-rates need to be averaged. For example, for the stochastic FHN neuron model, for repeat solution of the SDEs, there may be any number of action-potentials within a stimulus duration, $T$.

If the spike-count firing rate is used, the precision to which the output represents the input depends on the window duration, $T$. This is because the number of action-potentials during $T$ is limited to a small number of integers - i.e. the firing rate is a quantized representation of $I$. This is illustrated in Figs. 5(a) and 5(b).

For a shorter duration, the spike-count rate is a heavily quantized approximation to the ideal instantaneous rate (Fig. 5(a)). A large duration allows much higher precision (Fig. 5(b)). The presence of noise requires the spike-count rate to be used. This is a random variable - it can be seen that in this case there is some variance in the rate (Fig. 5(b)). 

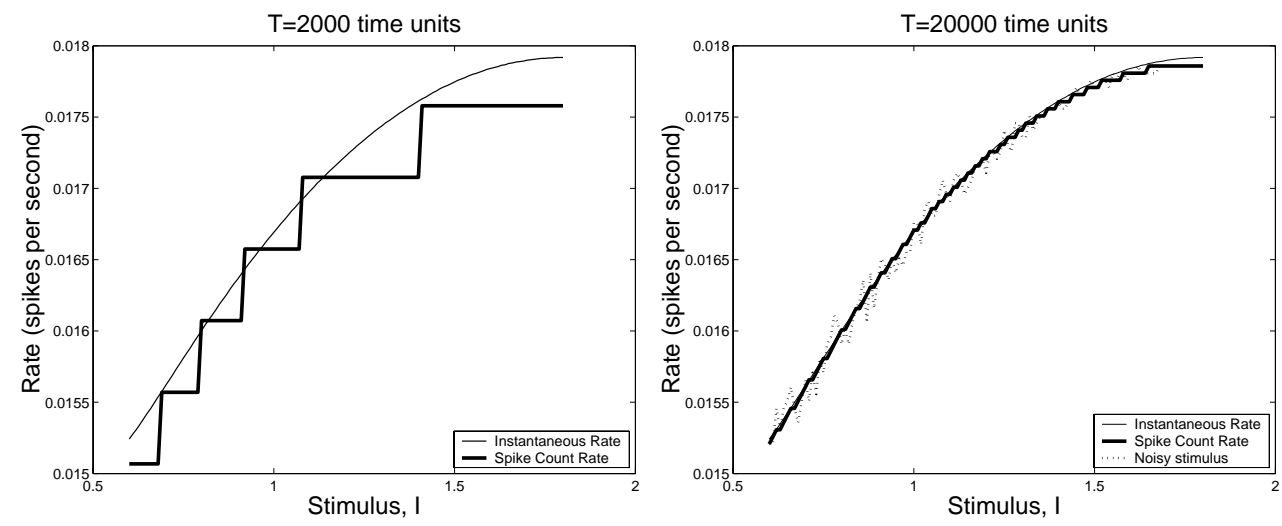

Figure 5. Tuning curves for the FHN neuron model. The units of $I$ are arbitrary.

\subsection{Discussion}

The results presented above illustrate the important points discussed in Sec. 2. In the absence of noise, it is theoretically possible for a neuron to uniquely encode, to infinite precision, any real value of current, $I$, by just two action-potentials. This would be achieved by precisely timing the interval between the two action-potentials. In other words, the time interval between the two action-potentials, $\Delta$, can be described as a continuous function of current, $I$, and therefore so can the firing rate, $R_{s}$. For the example with the FHN neuron model, we can write the firing rate as

$$
R_{s}=\frac{1}{\Delta}=f(I), \quad I \in[0.6,1.8],
$$

where $f(\cdot)$ is a continuous and strictly increasing function.

However, in the presence of noise, the ISI between any two action-potentials becomes a random variable. This means that encoding a stimulus to infinite precision by precise action-potentials timing is impossible. There are however neural mechanisms that do use precise timing (instantaneous rate) between two action-potentials - the noise in these mechanisms is sufficiently small that the variance in the firing rate allows for good precision. It is the level of the noise which determines whether instantaneous rate coding is sufficient.

For larger variability in ISIs, intuitively we would require more than two action-potentials in a given period of time to determine a mean firing rate. The larger the variability, the more action-potentials are required.

The crucial question arising from this is "how long a duration is required to obtain, on average, enough action-potentials to determine an accurate mean firing rate, that resolves a wide range of stimuli to a sufficient precision?"

For the absence of noise, although theoretically only two action-potentials are required, suppose an experimentalist is able to record from such a perfect neuron the number of action-potentials that occur during duration, $T$ - where $T$ is much larger than the ISI — but not the exact ISI. This scenario is much like the variable ISI case -if $T$ is small, a certain range of $I$ will provide the same number of action-potentials during $T$. In order to resolve to greater precision the firing rate for values of $I$ within that range, a longer $T$ is required.

Both this case, and the case of a randomly varying ISI can be considered in the quantization framework.

The answer to the posed question depends on the maximum and minimum number of action-potentials that can occur during $T$. The minimum number of action-potentials is zero. Suppose the maximum number is $N$. For a given stimulus intensity, $I$, let the number of action-potentials that occur during $T$ be $n$, where $n$ is an integer between 0 and $N$. This formulation means that the action-potential count is in fact an integer encoding of a real-valued parameter, $I$, or in other words, a quantization of $I$. The precision of the quantization increases with $N$, which in turn increases with the duration in which an action-potential count is made, $T$. 
For example, consider a current of $I=1.5$. During $T=2000$ seconds (Fig. 5(a)), there are 35 actionpotentials in the FHN model. However, there are also 35 action-potentials for $I$ between about 1.39 and 1.8 during this time, even though the instantaneous firing rate is slowly increasing.

If $T$ is increased by a factor of ten to $T=20000$ (Fig. 5(b)), we find that, if $I$ is discretized to a resolution of 0.01 , at $I=1.5$ there are 354 action-potentials, which only occurs for $I=1.48,1.49,1.50,1.51$.

As $T$ increases, the firing rate obtained using action-potential counting appears to get closer to the instantaneous rate. Of course, if $T=\infty$, we would expect both to coincide. The problem for a neuron though, is that the value of $T$ over which action-potentials might correspond to a fixed stimulus is likely to only be of short duration.

\subsubsection{Rate-Distortion Tradeoff}

The dynamics of the FHN equations define the tuning curve, and, therefore, the number of spikes during duration $T$ for a constant $I$; e.g. for $T=2000$ time-units (Fig. 5(a)):

- for $I \in[0.6,1.8]$, and $\beta=0$, possible values of $y$ are $\{30,31,32,33,34,35\}$;

- the thresholds that define which values of $I$ achieve each value of $y$ are about $\{0.69,0.8,0.92,1.08,1.41\}$.

Conventional quantization theory says we should define reproduction points, and a performance measure; we use mean square error (MSE) distortion, converted to SQNR. We also need to assume a probability distribution for the stimulus: in the absence of other knowledge, we take it to be uniform. This also requires assuming that the stimulus remains constant during duration, $T$. The calculated SQNR in dB is shown in Fig. 6. It can be seen, for example, that to achieve a distortion smaller than $0.0006(23 \mathrm{~dB}), T>24000$ is required.

The oscillatory behavior in Fig. 6 is due to the implied thresholds shifting position, while $N$ remains constant, with increasing $T$. Also, the number of states, $N$, for a given $T$, can sometimes decrease by unity, with an increasing $T$. Accuracy depends on the numerical resolution of $I$. We know the accuracy is sufficient if $N$ increases linearly with $T$. Further work will incorporate methods that smooth out this form of curve.

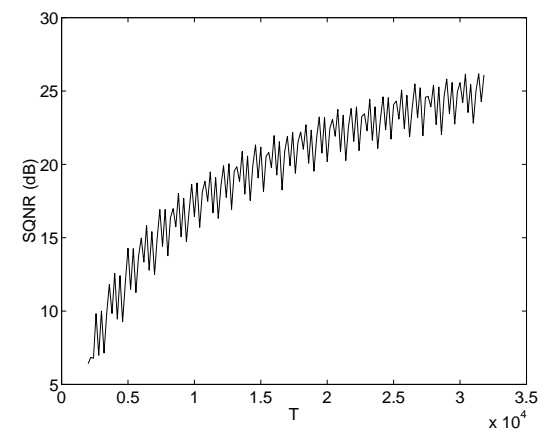

Figure 6. Tradeoff between action-potential counting time, $T$, and output SQNR, assuming the stimulus is uniformly distributed. This is equivalent to the information theoretic rate-distortion tradeoff. The units of $T$ are arbitrary.

\section{RELEVANCE FOR BIOMEDICAL ENGINEERING}

\subsection{Prosthetics}

As mentioned above, a phenomenon called suprathreshold stochastic resonance (SSR) can occur in neural models such as that in Fig. 2. This means that encoding performance is enhanced by the presence of random noise. This occurs due to the models' quantizing nature - unless the input SNR is extremely small, random noise can act like a dither signal to reduce distortion due to quantization. ${ }^{11}$ 
It has previously been shown that SSR can occur in arrays of FHN neuron models, ${ }^{10}$ as well as HodgkinHuxley and integrate-and-fire neuron models. ${ }^{14}$ When coupled with the fact that sensory neurons often are required to operate on signals with very small SNRs, this indicates that biological neural populations may exploit stochastic quantization effects to encode stimuli into action potentials. A prime candidate for further investigation into this topic is the auditory system. ${ }^{15}$

Due to much evidence supporting reduced stochastic neural activity in profoundly deaf humans, ${ }^{16}$ there have been a number of proposals to add random noise at the electro-biological interface in cochlear implants. These prosthetic devices restore hearing to profoundly deaf patients, via direct electrical stimulation of the cochlear nerve. The reason for these proposals is to try and mimic the natural random noise present in the form of Brownian motion, and spontaneous neural firing in a healthy inner ear. ${ }^{16,17}$ This randomness is missing in a deafened ear, and not present in current cochlear implants. In particular, it has also been proposed to replicate SSR effects in cochlear implant encoding. ${ }^{18}$ Under the assumption that the auditory system is optimized to cope with large levels of randomness, it is hypothesized that SSR effects may be utilized in the encoding of analog auditory signals (e.g. sound amplitude) into neural action potentials. If this turns out to have utility, there may well be other sensory systems for which future prosthetics also incorporate such effects.

\subsection{Biosensors}

The quantization model used in Fig. 2, where independent noise exists at the input to binary thresholding elements, is also applicable in non-neural situations. Such a situation can occur in sensor networks when local lossy compression is required prior to communication to a fusion hub. ${ }^{19}$ It has been demonstrated, in such a context, how the presence of iid noise in the values of threshold levels results in a reduction in the implementation complexity of optimal (or near-optimal) scalar quantizers. It is emphasized that this phenomenon relies on the assumption of independent noise, as well as low input SNRs. Such a situation may be applicable in artificial bio-sensors in biomedical applications, such as in sensors which measure biochemical quantities.

\section{CONCLUSIONS AND FUTURE WORK}

\subsection{Summary of Quantizer Approach to rate Coding}

Neural rate coding of an analog stimulus requires using a finite number of action potentials to represent that stimulus. Due to random noise, this means rate coding can be described as lossy compression, and in particular, a form of stochastic quantization.

Three timescales determine the rate-distortion performance of such a quantizer: (i) refractory times; (ii) duration of a counting window-'biophysical memory'; and (iii) stimulus correlation times. Together, these timescales define the achievable resolution of coding of a fixed stimulus. If biologically-relevant assumptions are made about these time scales, bounds on achievable performance can be found using quantization theory.

Alternatively, these timescales can be found experimentally: theoretical bounds can give an idea about how much lossy compression is too much to perceive changes in a signal. Complete analysis of this paradigm requires stochastic quantization theory. ${ }^{11,20}$ Provided a neuron has a fixed and well-defined threshold, then it is likely that some level of random noise is required for optimal performance. This is known as stochastic resonance. ${ }^{21}$

\subsection{Future Work}

This work has only just begun. New simulations will use more biologically realistic models. For a single neuron, accurate characterization of performance requires numerically calculating the probability of $N$ action-potentials during time $T$ for a given $I$. This is a very intensive computational task for the FHN equations.

Future work will also use standard quantization theory to extend and refine the results presented here. In particular, the affects of noise on neural quantization performance will be assessed. Preliminary simulations show that noise can act to shorten the duration $T$, over which an action-potential count needs to be made to achieve a certain average performance. This is analogous to dithering, in analog-to-digital converter circuits, or source coding algorithms. ${ }^{12}$

Furthermore, the arguments presented here will be refined and presented in a more precise manner, and applied to data obtained from real physiological neural experiments. 


\section{ACKNOWLEDGMENTS}

We would like to thank Anthony Burkitt and David Grayden of the Bionic Ear Institute, and The University of Melbourne, as well as David O'Carroll of The University of Adelaide for valuable discussions. Funding from the Australian Research Council (ARC) is gratefully acknowledged.

\section{REFERENCES}

1. P. Dayan and L. F. Abbott, Theoretical Neuroscience: Computational and Mathematical Modeling of Neural Systems, The MIT Press, 2001.

2. D. H. Johnson and W. Ray, "Optimal stimulus coding by neural populations using rate codes," Journal of Computational Neuroscience 16, pp. 129-138, 2004.

3. F. Rieke, D. Warland, R. de Ruyter van Steveninck, and W. Bialek, Spikes: Exploring the Neural Code, MIT Press, Cambridge, MA., 1997.

4. L. Kuhlmann, A. N. Burkitt, A. Paolini, and G. M. Clark, "Summation of spatiotemporal input patterns in leaky integrate-and-fire neurons: Applications to neurons in the cochlear nucleus receiving converging auditory nerve fiber input," Journal of Computational Neuroscience 12, pp. 55-73, 2002.

5. B. W. Knight, "Dynamics of encoding in a population of neurons," The Journal of General Physiology 59, pp. $734-766,1972$.

6. A. Gersho and R. M. Gray, Vector Quantization and Signal Compression, Kluwer Academic Publishers, 1992.

7. T. Berger and J. D. Gibson, "Lossy source coding," IEEE Transactions on Information Theory 44(6), pp. 2693-2723, 1998.

8. W. Bialek, M. DeWeese, F. Rieke, and D. Warland, "Bits and brains: Information flow in the nervous system," Physica A 200, pp. 581-593, 1993.

9. M. Bethge, D. Rotermund, and K. Pawelzik, "Optimal neural rate coding leads to bimodal firing rate distributions," Network: Computation in Neural Systems 14, pp. 303-319, 2003.

10. N. G. Stocks and R. Mannella, "Generic noise enhanced coding in neuronal arrays," Physical Review E 64, Art. No. 030902(R), 2001.

11. M. D. McDonnell, N. G. Stocks, C. E. M. Pearce, and D. Abbott, "Quantization in the presence of large amplitude threshold noise," Fluctuation and Noise Letters 5(3), pp. L457-L468, 2005.

12. R. A. Wannamaker, S. P. Lipshitz, and J. Vanderkooy, "Stochastic resonance as dithering," Physical Review E 61(1), pp. 233-236, 2000.

13. H. C. Tuckwell and R. Rodriguez, "Analytical and simulation results for stochastic Fitzhugh-Nagumo neurons and neural networks," Journal of Computational Neuroscience 5, pp. 91-113, 1998.

14. T. Hoch, G. Wenning, and K. Obermayer, "Optimal noise-aided signal transmission through populations of neurons," Physical Review E 68(3), Art. No. 011911, 2003.

15. F. Jaramillo and K. Wiesenfeld, "Mechanoelectrical transduction assisted by Brownian motion: a role for noise in the auditory system," Nature Neuroscience 1, pp. 384-388, 1998.

16. M. Chatterjee and M. E. Robert, "Noise enhances modulation sensitivity in cochlear implant listeners: Stochastic resonance in a prosthetic sensory system?, Journal of the Association for Research in Otolaryngology 2, pp. 159-171, 2001.

17. R. P. Morse and P. Roper, "Enhanced coding in a cochlear-implant model using additive noise: Aperiodic stochastic resonance with tuning," Physical Review E 61(5), pp. 5683-5692, 2000.

18. N. G. Stocks, D. Allingham, and R. P. Morse, "The application of suprathreshold stochastic resonance to cochlear implant coding," Fluctuation and Noise Letters 2(3), pp. L169-L181, 2002.

19. M. D. McDonnell, N. G. Stocks, and D. Abbott, "Complexity and distortion reduction in quantizers with independently random threshold levels." Submitted to IEEE Transactions on Signal Processing, 2006.

20. M. D. McDonnell, Theoretical Aspects of Stochastic Signal Quantisation and Suprathreshold Stochastic Resonance. PhD thesis, School of Electrical and Electronic Engineering, The University of Adelaide, Australia, 2006.

21. F. Moss, L. M. Ward, and W. G. Sannita, "Stochastic resonance and sensory information processing: a tutorial and review of application," Clinical Neurophysiology 115, pp. 267-281, 2004. 